\title{
Article \\ Detection of heavy metals in water using graphene quantum dots: an experimental and theoretical study
}

\author{
Lorenzo Gontrani $\mathbb{1}^{1 *}$, Olivia Pulci $\rrbracket^{2}$, Marilena Carbone $\rrbracket^{3}$, Roberto Pizzoferrato $\unrhd^{4, *}$, and Paolo Prosposito $\mathbb{1}^{4}$ \\ 1 Dipartimento di Chimica, Università di Roma "La Sapienza", P. le A. Moro 5, I-00185 Roma, Italy \\ 2 Dipartimento di Fisica, Università di Roma "Tor Vergata", Via della Ricerca Scientifica, I-00133 Roma, Italy; \\ olivia.pulci@uniroma2.it \\ 3 Dipartimento di Scienze e Tecnologie Chimiche, Università di Roma "Tor Vergata", Via della Ricerca \\ Scientifica, I-00133 Roma, Italy; marilena.carbone@uniroma2.it \\ 4 Dipartimento di Ingegneria Industriale, Università di Roma “Tor Vergata”, Viale degli Ingengeri, I-00133 \\ Roma, Italy; paolo.prosposito@uniroma2.it \\ * Correspondence:lorenzo.gontrani@uniroma1.it (L.G.); pizzoferrato@uniroma2.it (R.P.)
}

\begin{abstract}
In this work, we investigate by ab initio calculations and optical experiments the sensitivity of graphene quantum dots in their use as devices to measure the presence, and concentration, of heavy metals in water. We demonstrate that the quenching or enhancement in the optical response (absorption, emission) depends on the metallic ion considered. In particular, two test cases of opposite behaviour are considered: $\mathrm{Cd}^{2+}$, where we observe an increase in the optical response for increasing concentration, and $\mathrm{Pb}^{2+}$ whose emission spectra are quenched along the concentration rise. We envisage that quantum dots of graphene may be routinely used as cheap detectors to measure the degree of poisoning ions in water
\end{abstract}

Keywords: Graphene oxide; Quantum Dots; TD-DFT; Absorption, Quenching Effect, Concentration, Optical Response

\section{Introduction}

Heavy metals (HMs) have become a considerable factor of contamination of drinking water due to growing global industrialization, diffuse agricultural exploitation of land and, in some areas, natural occurrence with geological origin. Despite increasingly strict regulations and legislative actions, millions of people worldwide are exposed to high levels of HM intake via drinking water, while it is estimated that at least one million suffer from chronic poisoning in developing countries [1]. Moreover, the presence of HMs in surface waters and seawater contributes to the risks for human health through food chain accumulation. The highest level of hazard comes from species like cadmium, lead, chromium, arsenic, and mercury, which are systemic toxicants producing multiple organ damage and are classified as human carcinogens even at very low concentration [2]. For this reason, the Guidelines for Drinking-Water Quality by World Health Organization (WHO) [3] have set the threshold limits in the ppb range, e.g., $3 \mu \mathrm{g} / \mathrm{L}$ for $\mathrm{Cd}$ and $10 \mu \mathrm{g} / \mathrm{L}$

Citation: Lastname, F.; Lastname, F; Lastname, F. Title. Preprints 2021, 1, 0. https://doi.org/

Received:

Accepted:

Published:

Publisher's Note: MDPI stays neutral with regard to jurisdictional claims in published maps and institutional affiliations.

for $\mathrm{Pb}$. Determination of such low levels of concentration is generally performed by using well established laboratory methods including, but not limited to atomic high performance liquid chromatography (HPLC), absorption spectrophotometry (AAS), inductively coupled plasma mass spectrometry (ICP-MS) [4,5]. However, these techniques require expensive and heavy instrumentations, well-trained staff, and complex time-consuming procedures. These factors obviously hamper a diffuse monitoring of water quality, especially in developing countries. Nanomaterial-based optical sensing has recently demonstrated to be a promising candidate for the development of simple, friendly and affordable methods for $\mathrm{HM}$ monitoring. Based on colorimetric and fluorometric response, optical sensing is compatible with water solutions and is suitable for applications in a variety of portable systems, ranging from cheap paper-based detection to cost-effective handheld smartphone-based devices [6]. Graphene-like carbon-based nanoparticles, generally referred to as carbon dots (CDs), have recently been investigated for their sensitivity to HMs coupled with high stability and very low toxicity $[7,8]$. Sensitivity of CDs has been studied by several groups 
by recording the fluorescence quenching that occurs in the presence of HM ions in water solution [9]. Multiple and cross-sensitivity, however, limited the selective detection and discrimination of different species. We have recently investigated the sensing properties of carbon nanoparticles synthesized by cage-opening and unfolding C60 fullerene via a modified Hummers' reaction [10-12]. Sensitivity to HM ions in water was characterized through variations of both absorbance and fluorescence intensity, which enabled multiple and selective sensing of copper, lead, arsenic and cadmium in the same water solution. In the present contribution, we attempt at rationalizing the difference in such sensitivity using Density Functional Theory (DFT) and Time-Dependent Density Functional Theory (TD-DFT) calculations of the structures of ground and excited states of the nanoparticles and of their absorption and fluorescence spectra, with particular regard to the different solvent effect experienced by cadmium and lead cations quantum dots water dispersions that show the most pronounced concentration effect in their emission intensities.

\section{Materials and Methods}

\subsection{Experimental}

Synthesis of unfolded fullerene quantum dots (UFQDs) is described in more detail elsewhere $[10,11]$. Briefly, the Hummers' method consists in a strong oxidation of fullerene performed by using highly-oxidant agents $\left(\mathrm{NaNO}_{3}, \mathrm{KMnO}_{4}\right.$ and $\left.\mathrm{H}_{2} \mathrm{SO}_{4}\right)$ and a specific sequence of temperature variations that produce the opening of $\mathrm{C}_{60}$ cage. Subsequent purification trough dialysis resulted in a stable water dispersion of monodisperse UFQDs nanoparticles (see Fig.1) with lateral dimensions in the range from 2 to $4 \mathrm{~nm}$, as shown by dynamic light scattering (DLS), TEM and SEM images. Extensive characterization by NMR, Atomic Force Microscopy (AFM), X-Ray Photoelectron Spectroscopy (XPS), FourierTransform Infrared Spectroscopy (FTIR), X-Ray diffraction (XRD) and Raman spectroscopy confirmed that the nanoparticles had a main structure made of reduced graphene oxide (rGO) with the presence of a relatively low number of oxygen-containing functional groups $[10,11]$. The as-prepared water dispersion was diluted with deionized water to obtain a carbon nanoparticle concentration of $50 \mu \mathrm{M}$ that is therein referred to as UFQD stock solution. 


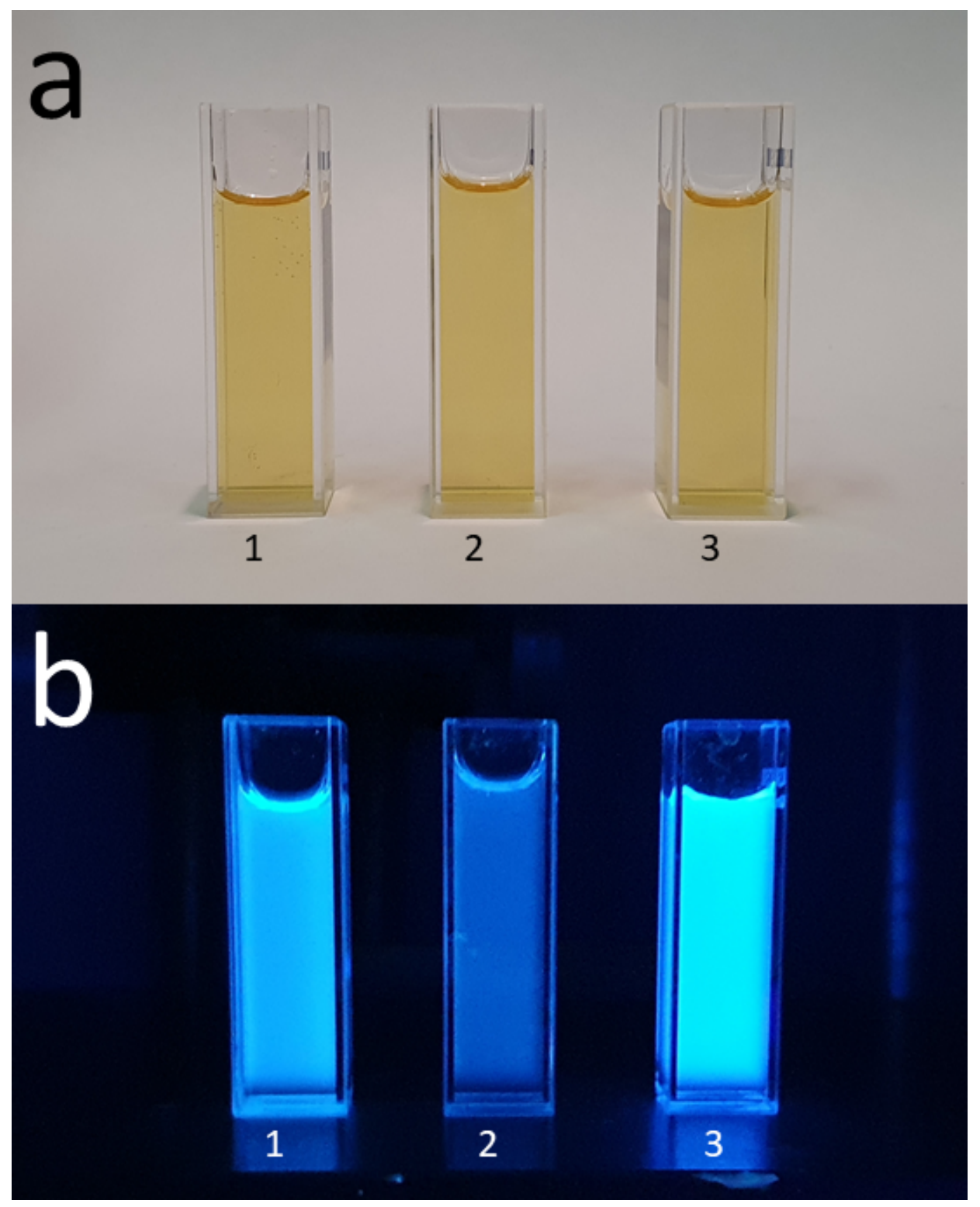

Figure 1. Photographs of UFQD sensing solution: (1) pure, (2) after addition of $\mathrm{Pb}^{2+}$ or (3) $\mathrm{Cd}^{2+}$ at a concentration of $100 \mu \mathrm{M}$ taken under visible light (a) and 365-nm UV light (b).

Experimental determination of sensitivity of UFQDs was performed by mixing $1 \mathrm{~mL}$ of UFQD stock solution with $1 \mathrm{~mL}$ of the HM-salt solution at the appropriate concentration in deionized water. After gentle stirring for $30 \mathrm{~s}$, absorbance and fluorescence spectra were recorded. UV-vis absorption spectra were measured with a Cary 50 spectrophotometer (Varian Inc., Palo Alto, CA, USA, USA) while fluorescence spectra were recorded with a laboratory setup for photoluminescence measurements [12]. The excitation wavelength $\lambda_{\text {exc }}=300 \mathrm{~nm}$ was used for fluorescence spectra with a spectral passband of $2 \mathrm{~nm}$ for both excitation and emission light.

\subsection{Computational}

We performed calculations on fragments of graphene, without and with metal ions, using DFT for geometry optimizations. Time-Dependent DFT (TD-DFT) was instead employed to determine the electronic excited state energies and the absorption spectra. Optical emission spectra where obtained as absorption spectra using the optimized excited state geometries. All the calculations were performed with the Gaussian 16 program [13]. 
Regarding the electronic transitions, the program outputs the energy/wavelength value of the transition as well as the "Oscillator Strength" $f$, an adimensional quantity that is related to the Dipole Strength D stored internally by the relation

$$
f_{i}=\frac{8 \pi^{2} \bar{v}_{i} m_{e} c}{3 h e^{2}} D_{i}
$$

In turn, the dipole strength is linked to $\bar{v}$, the excitation energy (in wavenumbers, i. e. the reciprocal of wavelength) and to $\epsilon_{i}^{\max }$, the intensity at the maximum (i. e. when the incident radiation $\bar{v}=\bar{v}_{i}$ ) by

$$
D_{i}=4 \cdot\left[\frac{3 \cdot 1000 \cdot \ln (10) \cdot h c}{32 \pi^{3} N}\right] \cdot \epsilon_{i}^{\max } \sqrt{\pi} \frac{\sigma}{\bar{v}_{i}}
$$

In these equations $\mathrm{N}$ is the Avogadro constant, $e$ is the electron charge, $\mathrm{m}_{e}$ the electron mass, $\mathrm{c}$ the speed of light, $\mathrm{h}$ the Planck constant and $\sigma$ is the standard deviation (bandwidth) equal to the half-width of the band when $\epsilon=\epsilon^{\max } / \mathrm{e}$ if the band shape is Gaussian. To obtain simulated UV-VIS spectra comparable to experimental ones, the software Gaussview [14] was used. The code assigns a Gaussian band shape

$$
\epsilon_{i}(\bar{v})=\epsilon_{i}^{\max } \exp \left[-\left(\frac{\bar{v}-\bar{v}_{i}}{\sigma}\right)\right]^{2}
$$

to every calculated absorption/emission transition and plots the convolution of all the bands. An Half-Width-Half-Height (HWHH) broadening of $0.333 \mathrm{eV}$ was used.

The starting point of the theoretical study consisted in a fragment of oxidized graphene that was obtained through fullerene unfolding (UFQD - see experimental section). The skeleton of this system is made up of five fused aromatic rings, identified by the letters A-E in Fig. 2, three carboxyl groups (two on E-ring and one on D-ring, respectively, and four $\mathrm{OH}$ groups, two phenol-like fragments and two benzyl $\left(\mathrm{CH}_{2} \mathrm{OH}\right)$ ones, totalling 55 atoms. The geometry of the fragment was first energy-minimized in vacuo using the density functional $\omega$-B97X-D with the split-valence basis set $6-31+G(d)$. The long-range corrected hybrid density functional chosen includes corrections for dispersion interactions and has proven effective in the calculation of geometries and excitation energies. [15] The optimization followed the Berny algorithm [16], with thresholds $4.5^{*} 10^{-5}$ and $3^{*} 10^{-5}$ Hartrees/Bohr for maximum and root-mean-square forces, respectively, on all the atoms. Since experimental data were gathered on nanoparticles water suspensions, the calculations were then extended to the solvated system, modeling the solvent with the Polarizable Continuum Model (PCM)[17]. According to this model, the charge distribution of the target molecule (or group of molecules/ions) identified as solute, is embedded into a given volume of space (the "molecular cavity") dug into an infinite continuum dielectric (the solvent) characterized by specific macroscopic properties (density, refractive index, dielectric permittivity $\epsilon$, etc.). The field produced by the solute charges into the exterior volume interacts with the solvent itself (described as a polarizable dielectric), and leads to the establishment of an apparent surface charge distribution $\zeta$ on the cavity surface. A new field, called reaction field, is produced in response to such charge and it can ultimately modify the previous solute charge distribution. The combination of solute and solvent reaction fields can be analytically treated, so that a final state in which both distributions are mutually equilibrated is obtained. Within the quantum mechanical framework, the interactions between solvent and solute are modeled through specific (perturbation or reaction) operators of the solute Hamiltonian.

The Schrödinger equation describing the system becomes

$$
\left(H^{0}+V^{\text {Reac }}\right)|\psi\rangle=E|\psi\rangle
$$

where $\mathrm{H}^{0}$ is the Hamiltonian in the absence of the solvent. 
The solvent operator $\mathrm{V}^{\text {Reac }}$ acting on $\psi$ depends on the surface apparent charge $\zeta$, and ultimately, as said before, on the solute charge distribution (i.e. the solute wavefunction $\psi$ ). This mutual interactions between $\psi$ and $V^{\text {Reac }}$ assures that the solution of Eq.(5) represents an equilibrated solute-solvent system. The calculations were carried out using Gaussian software [13] that fully implements the PCM method [17], choosing water as solvent (static dielectric permittivity $\epsilon=78.3553$, permittivity at infinite frequency $\epsilon(\infty)=1.7778$ ).

\section{Results and Discussion}

The experimental absorption and emission spectra of water dispersions of UFQDs in the presence of $\mathrm{Pb}^{2+}$ and $\mathrm{Cd}^{2+}$ at different ion concentrations are reported in Fig. 3 and 4 , respectively, and are labelled as a (absorption) and b (emission). The choice of these two cations was suggested by the remarkable intensity trend with concentration observed in their absorption and emission spectra that could be profitably exploited in purposely designed sensors. Namely, $\mathrm{Cd}$ and $\mathrm{Pb}$ give opposite trends in the emission spectra with increasing ion concentration in water (Cd: enhancement, Pb quenching). See Fig. 1 and $[10,11]$. On the contrary, the effect of concentration on absorption spectra is limited to an intensity increase with the concentration for $\mathrm{Pb}^{2+}$ cation solutions below $300 \mathrm{~nm}$, while is almost absent in the other cation solutions. In each picture, the spectra measured for the water dispersions of "bare" (non coordinated to metal, black line) UFQDs and cationcoordinated system at different metal to graphene fragment molar ratios $\left(\mathrm{R}_{M}\right.$, pink to blue lines) are piled up (the arrows in the figures are a guide for the eyes). Regarding the emission spectra, it can be noticed that all the dispersions containing the same cation share the same wavelength $(\lambda)$ for the peak maximum, that is $455 \mathrm{~nm}$ for $\mathrm{Pb}^{2+}$ and $450 \mathrm{~nm}$ for $\mathrm{Cd}^{2+}$, whereas the absorption spectra do not show peaks in the 250-500 $\mathrm{nm} \lambda$ range, and only a faint shoulder at $305 \mathrm{~nm}$ can be pointed out in both systems (dashed-line circle in Fig. 3-4). The observed fluorescence red-shift is therefore about $150 \mathrm{~nm}$ for both systems. The calculation of the absorption and emission spectra was accomplished according to the protocol described in Section 2. In the first step the optimized structure of the oxidized graphene fragment in water solution (modelled by PCM) was obtained. The relative geometry is reported in Fig.2 and shows a sizable curvature if compared with the planar periodic arrangement of graphene sheets without substituents.

Such bending is observed both in vacuo and in the PCM model. Once the relaxed structure was obtained, a linear response TD-DFT calculation of the system was accomplished, thus obtaining the vertical transitions to 20 excited states both in vacuo and in PCM solution.

The UFQD fragment (host) was then further modified in order to allow for the interaction with a cation (guest). Given that the cations considered in this study are both divalent (charge +2), two carboxylic groups (one in ring E, one in ring D) were deprotonated to finally obtain a neutral system. In particular, $\mathrm{Cd}$ and $\mathrm{Pb}$ cations were used to build the complex. These models, that will be labeled "UFQCD-Cd" (or Pb) hereafter, were treated in a similar way (geometry optimization and TD-DFT) as the non-complexed system, with the additional excited state relaxation after the absorption step. In this procedure, the potential energy surface (PES) of the first excited state was scanned in search of a local miminum and for every sampled point a TD-DFT calculation with 5 transition was performed. Given the complexity of the calculation, characterized by very flat potential energy surfaces, the optimizer search step (in terms of change in geometrical coordinates) was greatly reduced from the default values up to 0.05 Bohrs and 0.05 radians. If the changes from step to step are not very drastic, the chances of swapping between the target excited state and other (wrong) excited states surfaces of similar energy is lower and the overall time needed for the optimization decreases, in spite of the smaller step adopted.[18]. This way, the optimized structures could be obtained, satisfying the same convergence criteria as in the ground state. A larger TD-DFT calculation with 20 transitions was then run on the optimized excited state geometry. The calculated vertical transitions using this relaxed geometry correspond to the emission wavelengths and were calculated in PCM medium 


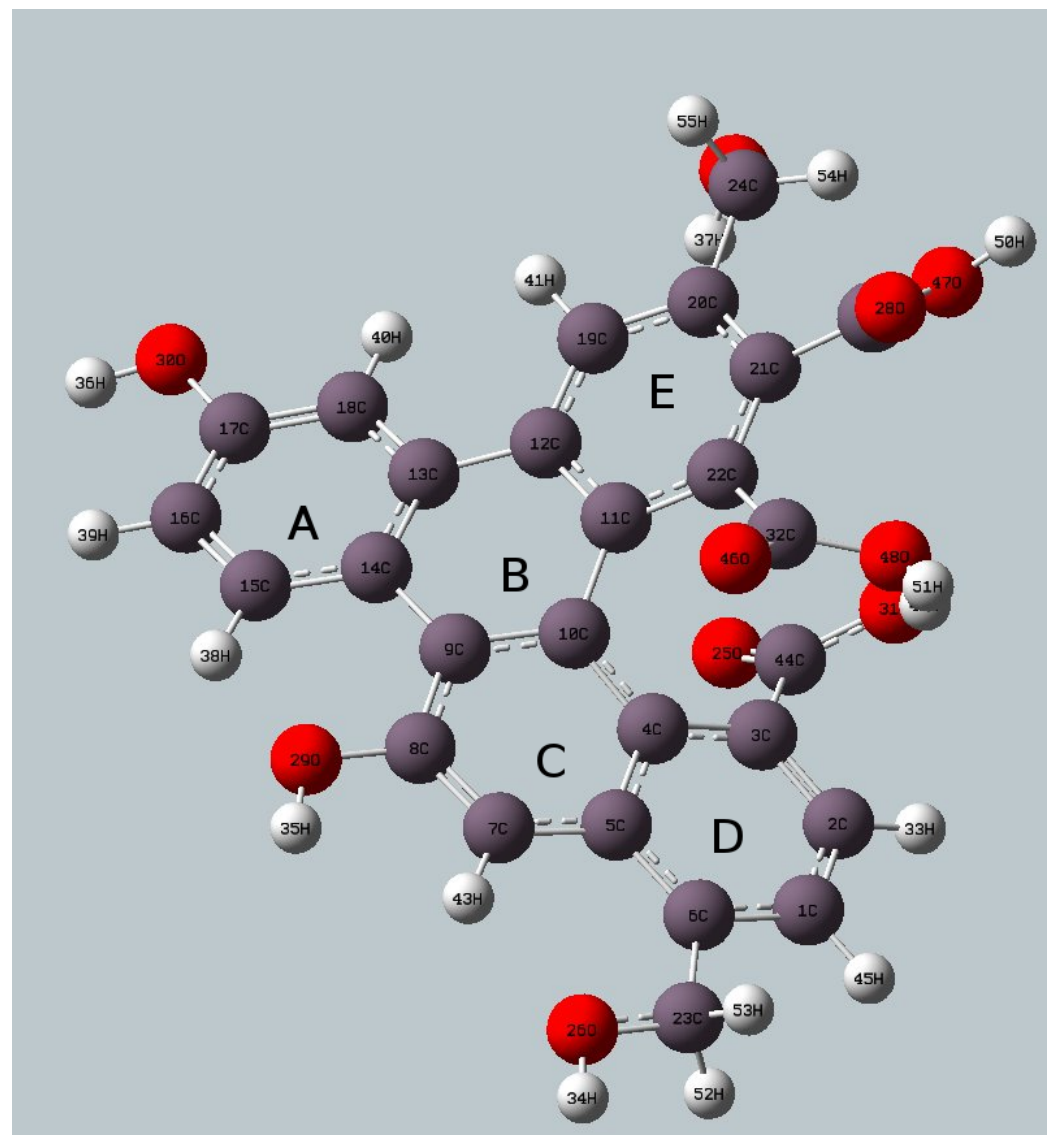

Figure 2. Optimized geometry of bare UFQD at $\omega$-B97XD/6-31+G(d) level

also for UFQD fragment not complexed with the cation for comparison. [18]. Finally, a further group of models was generated - UFQCD-Cd-WAT and UFQCD-Pb-WAT - where the solvent effect was taken into account explicitly, by adding three water molecules in the solvation shell of the ion. These models will be identified as "DILUTED models" as opposed to the "CONCENTRATED" ones. 

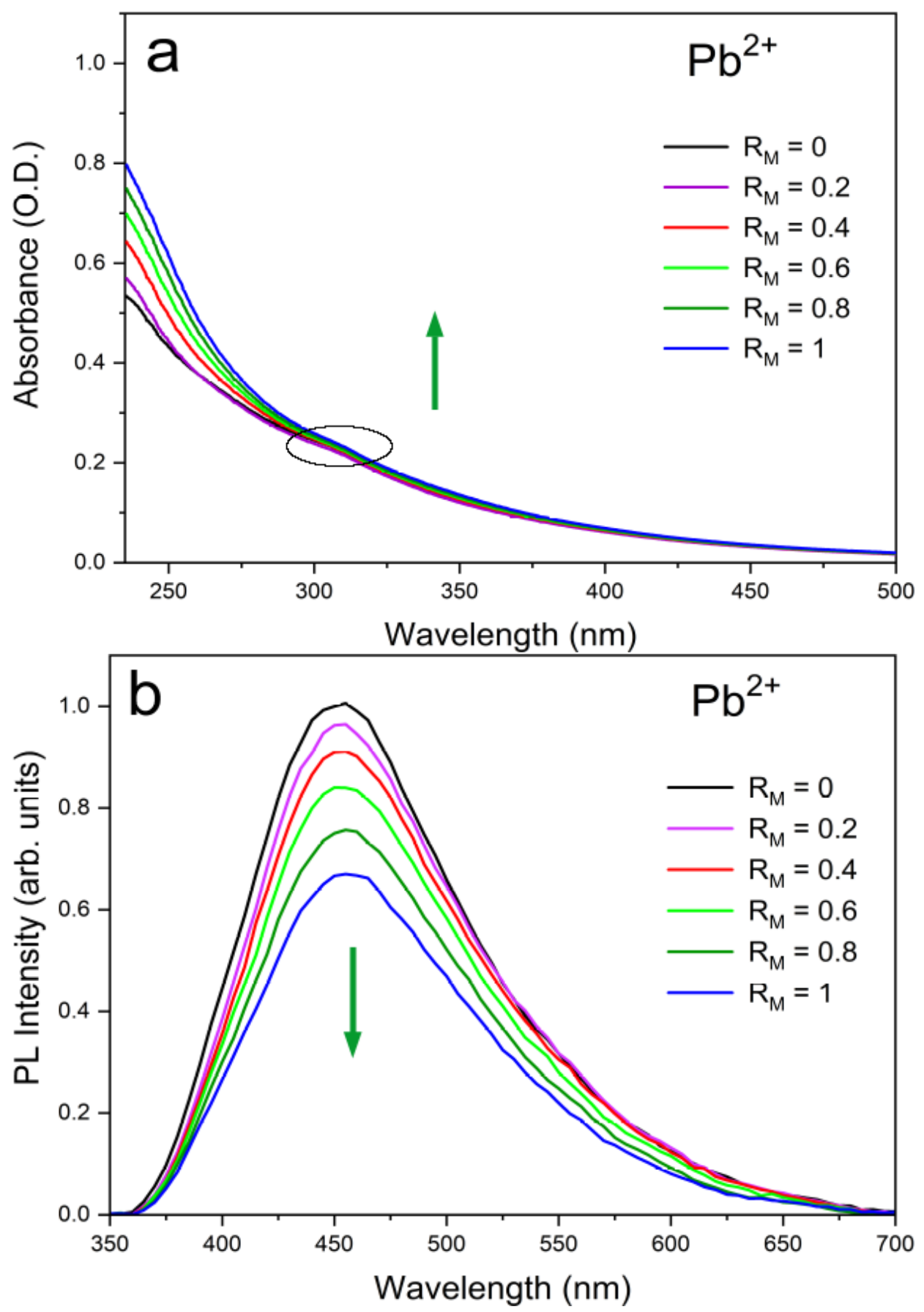

Figure 3. Experimental optical absorption (a) and emission (b) spectra of the aqueous suspension of UFQDs in the presence of $\mathrm{Pb}^{2+}$ at different values of the ratio of the respective molar concentrations $\mathrm{RM}=\left[\mathrm{Pb}^{2+}\right] /[\mathrm{UFQD}]$ 

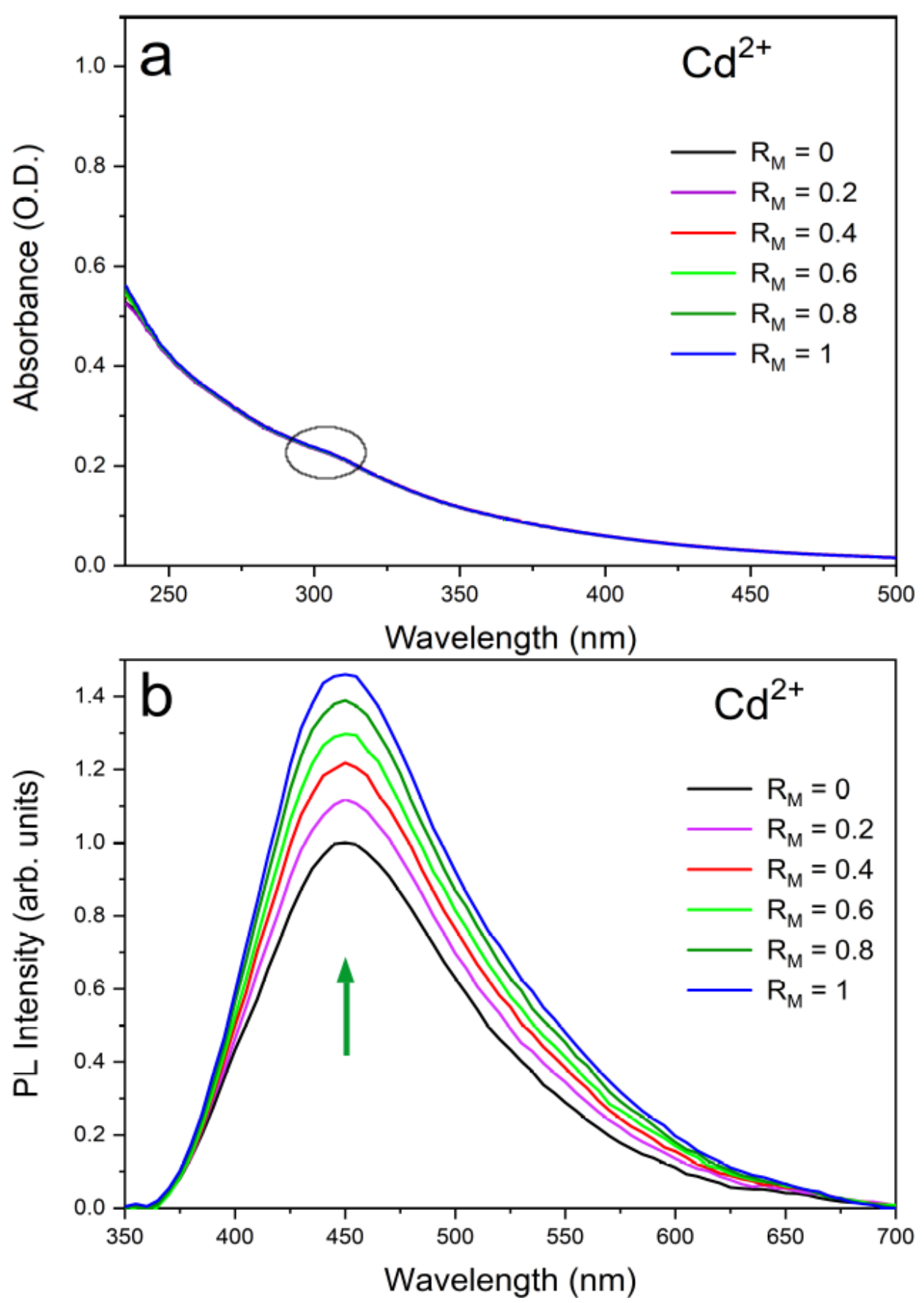

Figure 4. Experimental optical absorption (a) and emission (b) spectra of the aqueous suspension of UFQDs in the presence of $\mathrm{Cd}^{2+}$ at different values of the ratio of the respective molar concentrations $\mathrm{RM}=\left[\mathrm{Cd}^{2+}\right] /[\mathrm{UFQD}]$.

The values of the highest wavelength in the calculated absorption and emission spectra are collected in table 1. The calculated red-shifts (Stokes shifts) between absorption and emission fall in the 100-120 nm range, and comply satisfactorily with experimental data, also considering the uncertainty in the position of the shoulder in the absorption spectra. Moreover, for every calculated transition, the coefficients (c) of the TDDFT expansion matrix containing one-electron promotions could be used to "assign" the transition, according to the following formula, valid for closed-shell configurations (like the systems considered):

$$
\% \text { contrib }=c^{2} * 2 * 100
$$


that yields the percent contribution of a given transition between ground state orbitals (e. g. $\mathrm{HOMO} \rightarrow$ LUMO) to the excited state [19]. It should be noticed that the emission transitions have "purer" HOMO $\rightarrow$ LUMO character in any case, whereas the absorption lines of the ioncontaining system are more contaminated by other transitions (mainly HOMO- $1 \rightarrow$ LUMO and $\mathrm{HOMO} \rightarrow \mathrm{LUMO}+1$ ), and the oscillator strength values of the transitions increase markedly. The increase of $\mathrm{HOMO} \rightarrow$ LUMO character is probably brought about by the expunction of some states having energy degeneracy from the TDDFT interaction matrix upon the structure relaxation, and/or by the relative transitions turning to forbidden.

Table 1. Energy, Wavelength, Oscillator Strengths and percent contributions of HOMO-LUMO transition of the absorption and emission spectrum of graphene-ion systems. The letter $\mathrm{W}$ refers to model with explicit waters (diluted)

\begin{tabular}{cccccc}
\hline System & $\mathbf{E}(\mathbf{e V})$ & $\lambda \mathbf{( n m )}$ & Osc. Str. & \% HOMO-LUMO & Concentration \\
\hline Absorption & & & & & \\
UFQD & 2.98 & 416.23 & 0.1793 & 90.92 & No ion \\
UFQD-Pb & 3.68 & 337.24 & 0.1526 & 55.00 & Concentrated \\
& 3.73 & 332.26 & 0.1829 & 27.51 & $"$ \\
UFQD-PbW & 3.69 & 336.06 & 0.0397 & 5.26 & Diluted \\
& 3.78 & 328.44 & 0.1307 & 79.39 & $"$ \\
UFQD-Cd & 3.69 & 335.83 & 0.1196 & 21.43 & Concentrated \\
& 3.75 & 331.07 & 0.2606 & 69.20 & $"$ \\
UFQD-CdW & 3.69 & 336.33 & 0.0331 & 9.05 & Diluted \\
& 3.77 & 328.77 & 0.1303 & 77.80 & $"$ \\
\hline Emission & & & & & \\
UFQD & 2.80 & 443.46 & 0.4264 & 94.89 & No ion \\
UFQD-Pb & 2.81 & 440.89 & 0.4238 & 95.30 & Concentrated \\
UFQD-PbW & 2.76 & 448.70 & 0.4428 & 94.66 & Diluted \\
UFQD-Cd & 2.82 & 440.14 & 0.4743 & 95.24 & Concentrated \\
UFQD-CdW & 2.77 & 447.63 & 0.4457 & 94.78 & Diluted \\
\hline
\end{tabular}

The data reported in table 1 appear to be rather in agreement with most experimental trends. Actually, the absorption wavelength of the largest $\lambda$ transition remains almost constant when passing from "diluted" to "concentrated" models in both ions' spectra, and are not far from the wavelength values of the shoulder observed. Yet, the results are not straightforward, since the second-lowest transition is calculated to have the predominant HOMO-LUMO character in $\mathrm{Cd}^{2+}$ but not in $\mathrm{Pb}^{2+}$, so that a small shift (i. e. from 328 to $337 \mathrm{~nm}$ ) would be predicted in this case. Overall, bearing in mind that the experimental pattern results from the convolution of bands of non-infinitesimal width, the analysis should not be focused on the single lines only, but should consider the entire peak(shape), and in this case the agreement would be regarded as satisfactory (left panels of Fig. $5,6)$. The oscillator strength values, in turn, appear to rise a bit for $\mathrm{Pb}^{2+}$ from 0.13 to $0.15 / 0.18$ units, but this seems to happen for the other ion as well, opposite to the experimental finding. On the contrary, the emission data trends, where the concentration effect in the experimental patterns is much more evident, are reproduced quite correctly. Indeed, along the concentration increase, the opposite behaviour of the systems can be observed: fluorescence is quenched (Osc. Str. from 0.44 to 0.42 ) in presence of $\mathrm{Pb}^{2+}$ ions, while the signal is enhanced (from 0.45 to 0.47 ) with $\mathrm{Cd}^{2+}$. An evident discrepancy with experimental data, though, was found in the calculation of the vertical absorption of the UFQD (PCM) solution without ions, that is wrongly predicted at $416 \mathrm{~nm}$. The theoretical patterns obtained from the broadening of the calculated transition lines (see Materials and Methods section) are shown in Fig. $5\left(\mathrm{~Pb}^{2+}\right)$ and $6\left(\mathrm{Cd}^{2+}\right)$, in the $320-350 \mathrm{~nm}$ range for absorption (left panel) and in the 430-460 range (right panel) for emission, covering this way the lowest energy part of both phenomena. In order to tentatively explain the observed trends, the HOMO and LUMO orbital densities of both absorption and emission 
ground states, as well as the total electron densities of ground and excited states and their differences were analyzed. The difference between LUMO and HOMO surfaces (reported in Fig.7), as well as the data reported in Table 1 suggest that the process can be classified as a $\pi \rightarrow \pi^{*}$ transition occurring within $\mathrm{sp}^{2}$-carbon domains of the graphene-like defective lattice. Interestingly, the HOMO and LUMO densities do not show large isovalues around the ion, but the latter is capable of inducing a different deformation of the conjugated $\pi$ system nearby. In fact, $\mathrm{Pb}^{2+}$ establishes three interactions with negatively charged polar groups of the graphene nanoparticles, one with $\mathrm{D}$ ring $\mathrm{COO}^{-}$group and two with $\mathrm{E}$ ring ( $\mathrm{COOH}$ and $\mathrm{COO}^{-}$groups), whereas $\mathrm{Cd}^{2+}$ forms only two such interactions, since the E-ring $\mathrm{COOH}$ moiety interacts with the alcohol $\mathrm{CH}_{2} \mathrm{OH}$ group of ring $\mathrm{E}$ through a hydrogen bond. The latter interaction pattern results in a small uplifting (See Fig. 8, left panel, cyan) of the A-B-E rings portion (the out-of-plane dihedral angle between B and E ring decreases about 5 degrees) that ultimately causes a larger electron transfer towards $A$ and $B$ rings (from the ion towards the left in Fig.7). This observation is confirmed by the variation of the total dipole moment calculated on the whole electron density of the excited states, that can be both largely described as ground state LUMOs (see table 1), which increases by 1.22 Debye in $\mathrm{Cd}^{2+}$ and by 1.02 in $\mathrm{Pb}^{2+}$ with respect to the ground state. Such variation goes hand in hand with the (small) difference in the oscillator strengths calculated. When the UFQDs are "diluted", as in the model with three explicit waters, the water cap surrounding the nanoparticle (from the ion side) prevents the $\mathrm{COOH} \cdots \mathrm{CH}_{2} \mathrm{OH}$ hydrogen bonding and the ring structures are largely superimposable, with the only small structural differences confined to the ion-water interactions. This geometrical arrangement is shown in Fig. 8, where the structures of hydrated and non-hydrated models for both ions are superimposed. The main consequences of the structural coincidence of cromophores are that the two LUMO surfaces are substantially equivalent, the oscillator strengths are almost equal and the dipole moment variations are negligible ( 0.0014 and 0.058 Debye in $\mathrm{Cd}^{2+}$ and $\mathrm{Pb}^{2+}$, respectively).

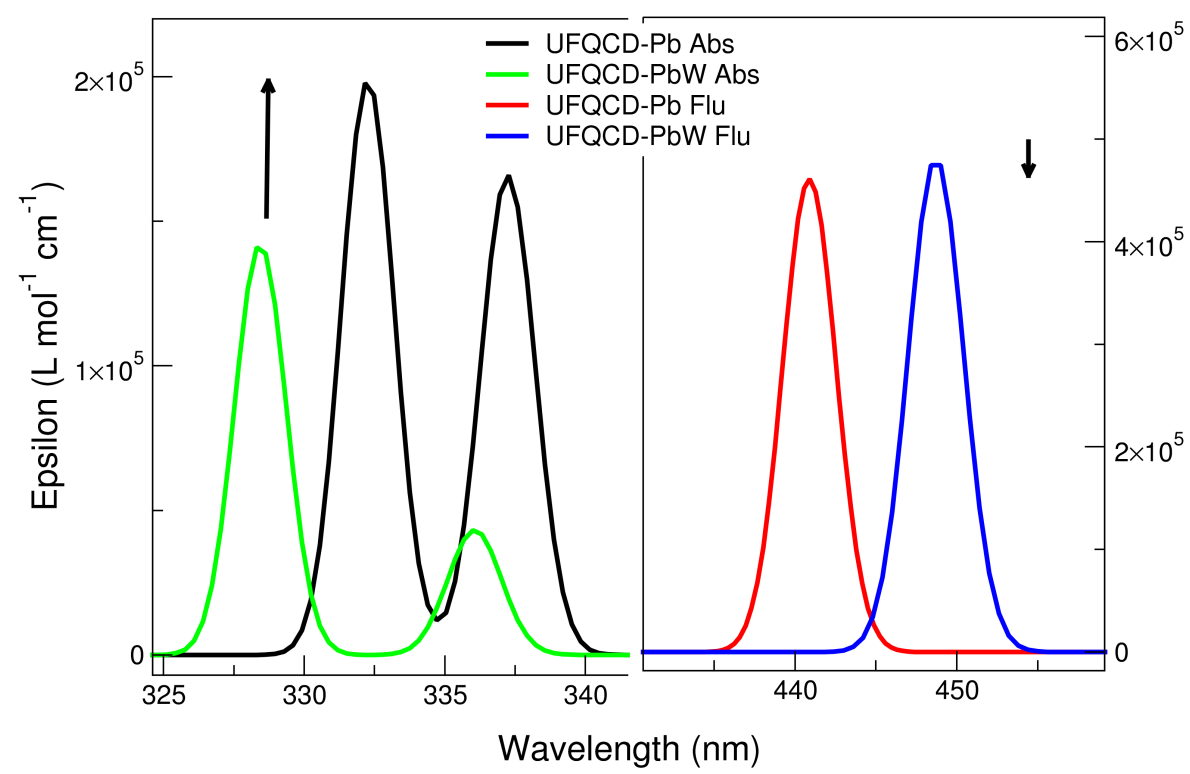

Figure 5. UFQD-Pb absorption (left) and emission (right) theoretical spectra. 


\section{Conclusions}

In this paper we propose a theoretical/computational explanation of the intensity features observed in the absorption and emission spectra of water dispersions of Unfolded Fullerene Quantum Dots in the presence of metal ions. The use of UFQDs within a spectrophotometric protocol for the detection of small concentration of polluting heavy metal ions is a very effective and rather low-cost method of water analysis. The simulation protocol reported appears to be appropriate to reproduce, at least to a qualitative degree, the quenching effect of the fluorescence that occurs when $\mathrm{Pb}^{2+}$ concentration in the system increases, and the intensity rise experienced by $\mathrm{Cd}^{2+}$ dispersions with increasing cation content. The main reason leading to the opposite behaviours appears to reside in the different conformations of two rings of the graphene cromophore. In $\mathrm{Cd}^{2+}$ system, the cation is predicted to be less solvated (coordinated) by the carboxy polar groups than $\mathrm{Pb}^{2+}$ system, thus permitting the establishment of an intermolecular hydrogen bond with a alcohol group further apart, that pushes the surrounding carbon rings. This geometrical arrangement favours a larger HOMO-LUMO electron transfer and the increase of dipole moment increase and transition oscillator strength, in compliance with the higher fluorescence intensity. When the cation is surrounded by water molecules (dilution), the latter mechanism does not operate and the two systems are predicted to have a very similar behaviour.

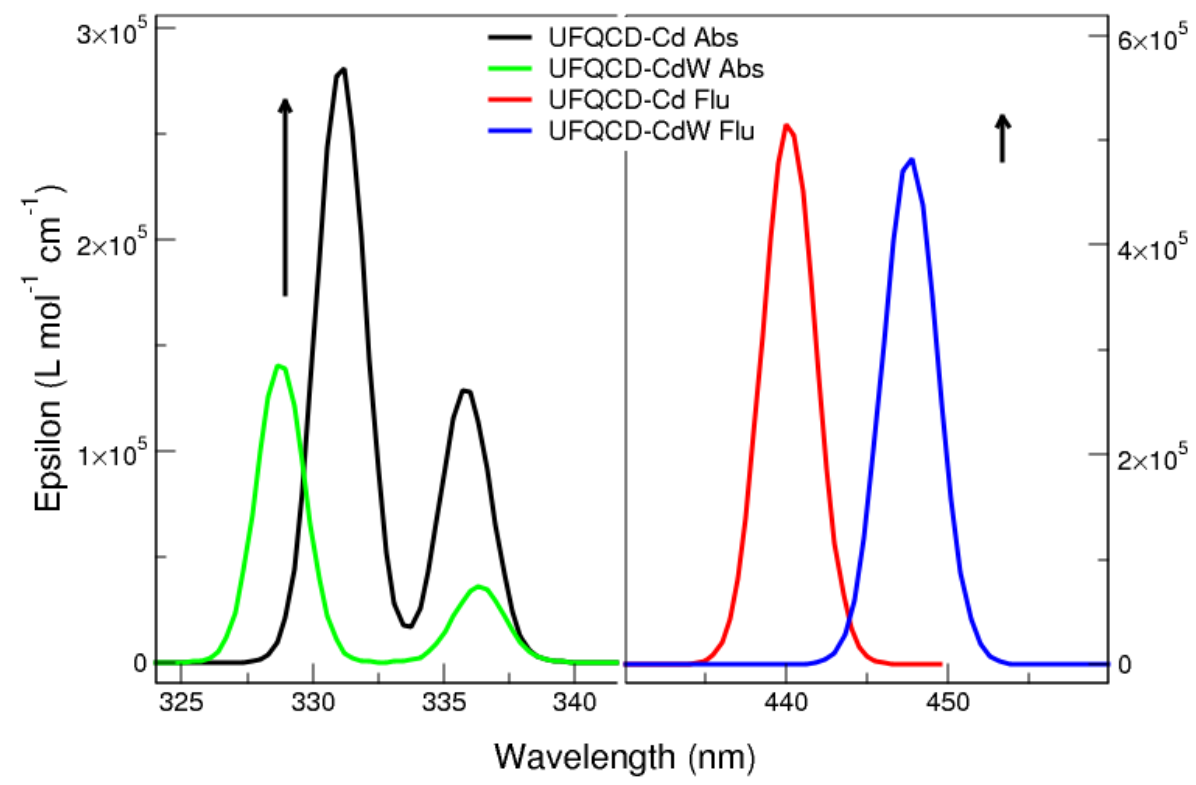

Figure 6. UFQD-Cd absorption (left panel) and UFQD-Cd emission (right panel) theoretical spectra 


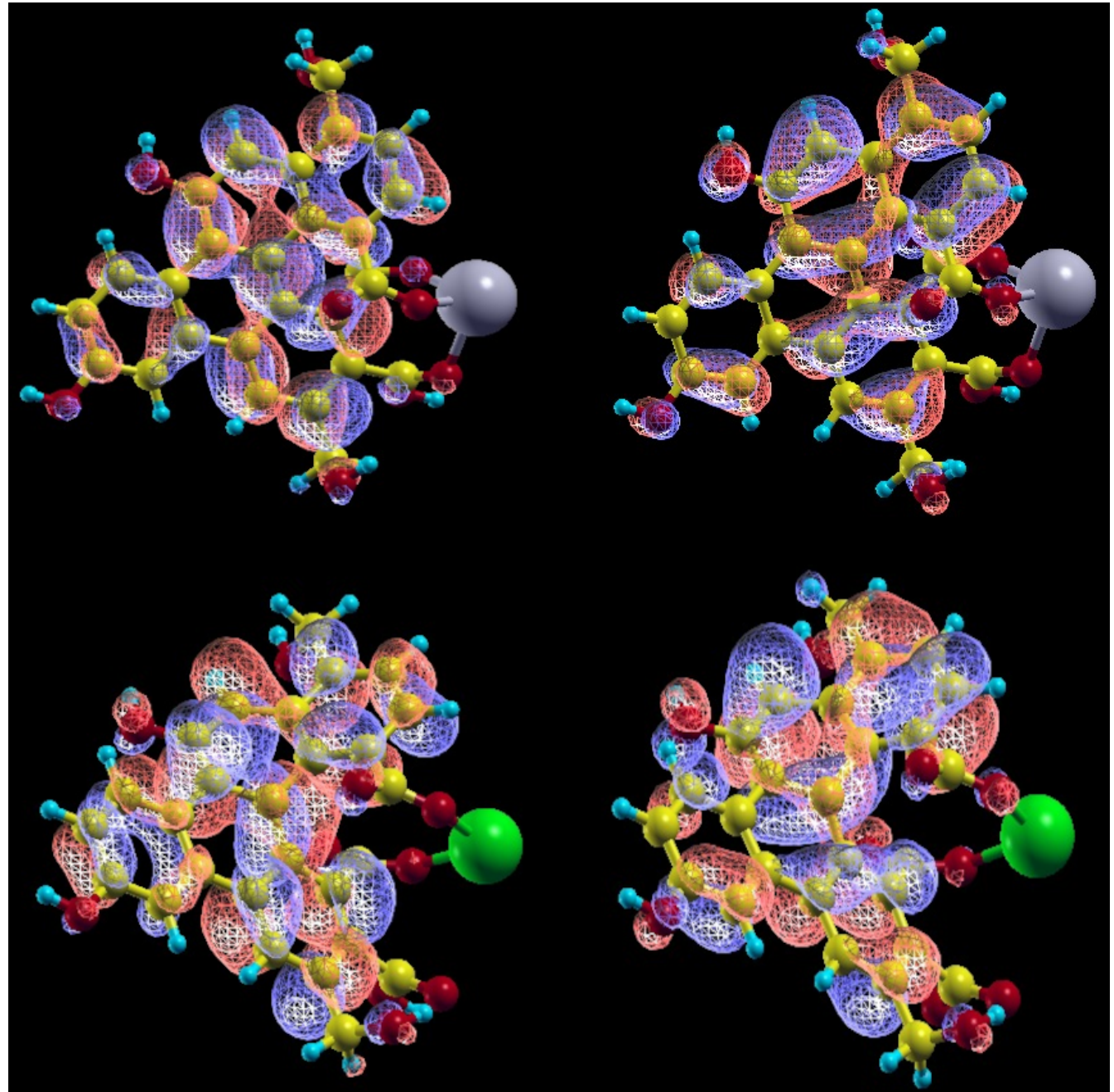

Figure 7. HOMO and LUMO density surfaces of UFQD-Pb (top) and UFQD-Pb (bottom). HOMO: right panel, LUMO: left panel

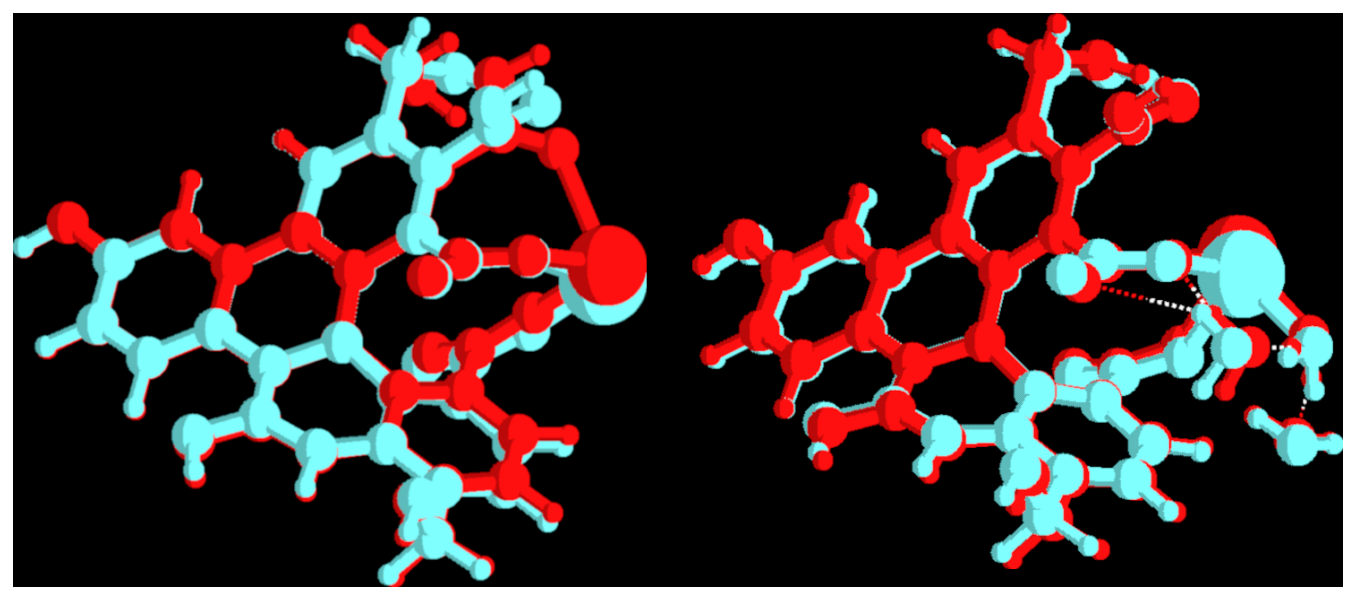

Figure 8. Overlay of UFQD-Cd/UFQD-Pb (left panel) and of UFQD-CdW/ UFQD-PbW structures (right panel). $\mathrm{Pb}$ : red; $\mathrm{Cd}$ : cyan 
Author Contributions: Conceptualization, L.G. , O.P. and R. P.; methodology, L. G. and O. P.; software, L.G.; validation, M.C., P.P., O.P. and R.P.; formal analysis, M.C. and P.P.; investigation, L.G.; resources, P.P.; data curation, R.P.; writing—original draft preparation, L.G., R.P.; writing-review and editing, L.G and O.P.; visualization, P.P and M.C.; supervision, M.C. and O.P.; project administration, P.P.; funding acquisition, P.P. and O.P. All authors have read and agreed to the published version of the manuscript.

Funding: L.G. acknowledges support from Regione Lazio, through Progetto di Ricerca 85-2017-15125 according to L. R. 13/08. CPU computing time was granted by CINECA HPC center and by the "Departments of Excellence-2018" Program (Dipartimenti di Eccellenza) of the Italian Ministry of Research, DIBAF-Department of University of Tuscia, Project "Landscape 4.0 - food, wellbeing and environment" (Prof. Nico Sanna). O.P. acknowledges financial funding from the EU MSCA-RISE project DiSeTCom (GA 823728).

\section{Acknowledgments:}

Conflicts of Interest: The authors declare no conflict of interest.

\begin{tabular}{|c|c|}
\hline \multicolumn{2}{|c|}{ Abbreviations } \\
\hline \multicolumn{2}{|c|}{$\begin{array}{l}\text { The following abbreviations are used in this manuscript } \\
\text { the }\end{array}$} \\
\hline DFT & Density Functional Theory \\
\hline TDDFT & Time Dependent Density Functional Theory \\
\hline PCM & Polarizable Continuum Model \\
\hline UFQD & Unfolded Fullerene Quantum Dots \\
\hline $\mathrm{HOMO}$ & Highest Occupied Molecular Orbital \\
\hline LUMO & Lowest Unoccupied Molecular Orbital \\
\hline
\end{tabular}

\section{References}

1. Fernández-Luqueño, F.; López-Valdez, F.; Gamero-Melo, P.; Luna-Suárez, S.; Aguilera-González, E.; Martínez, A.; GarcíaGuillermo, M.; Hernández-Martínez, G.; Herrera-Mendoza, R.; Álvarez Garza, M.; Pérez-Velázquez, I. Heavy metal pollution in drinking water - a global risk for human health: A review. African Journal of Environmental Science and Technology 2013, 7, 567-584.

2. Tchounwou, P.B.; Yedjou, C.G.; Patlolla, A.K.; Sutton, D.J., Heavy Metal Toxicity and the Environment. In Molecular, Clinical and Environmental Toxicology: Volume 3: Environmental Toxicology; Luch, A., Ed.; Springer Basel: Basel, 2012; pp. 133-164. doi:10.1007/978-3-7643-8340-4_6.

3. Gordon, B.; Callan, P. Guidelines for Drinking-Water Quality Fourth Edition WHO Library Cataloguing-in-Publication Data 2011.

4. Ressalan, S.; Chauhan, R.; Goswami, A.; Purohit, D. Review of Spectrophotometric Methods for Determination of Chromium. Reviews in Analytical Chemistry 1997, 16, 69-171. doi:doi:10.1515/REVAC.1997.16.2.69.

5. Ackerman, A.H.; Creed, P.A.; Parks, A.N.; Fricke, M.W.; Schwegel, C.A.; Creed, J.T.; Heitkemper, D.T.; Vela, N.P. Comparison of a Chemical and Enzymatic Extraction of Arsenic from Rice and an Assessment of the Arsenic Absorption from Contaminated Water by Cooked Rice. Environmental Science \& Technology 2005, 39, 5241-5246, [https://doi.org/10.1021/es048150n]. PMID: 16082952, doi:10.1021/es048150n.

6. Liu, T.; Wang, W.; Jian, D.; Li, J.; Ding, H.; Yi, D.; Liu, F.; Wang, S. Quantitative remote and on-site Hg2+ detection using the handheld smartphone based optical fiber fluorescence sensor (SOFFS). Sensors and Actuators B: Chemical 2019, $301,127168$. doi:https:/ / doi.org/10.1016/j.snb.2019.127168.

7. Long, C.; Jiang, Z.; Shangguan, J.; Qing, T.; Zhang, P.; Feng, B. Applications of carbon dots in environmental pollution control: A review. Chemical Engineering Journal 2021, 406, 126848. doi:https://doi.org/10.1016/j.cej.2020.126848.

8. Xu, D.; Lin, Q.; Chang, H.T. Recent Advances and Sensing Applications of Carbon Dots. Small Methods 2020, 4, 1900387, [https:/ / onlinelibrary.wiley.com/doi/pdf/10.1002/smtd.201900387]. doi:https://doi.org/10.1002/smtd.201900387.

9. Shen, J.; Zhu, Y.; Yang, X.; Li, C. Graphene quantum dots: emergent nanolights for bioimaging, sensors, catalysis and photovoltaic devices. Chem. Commun. 2012, 48, 3686-3699. doi:10.1039/C2CC00110A.

10. Ciotta, E.; Paoloni, S.; Richetta, M.; Prosposito, P.; Tagliatesta, P.; Lorecchio, C.; Venditti, I.; Fratoddi, I.; Casciardi, S.; Pizzoferrato, R. Sensitivity to Heavy-Metal Ions of Unfolded Fullerene Quantum Dots. Sensors 2017, 17. doi:10.3390/s17112614.

11. Ciotta, E.; Prosposito, P.; Tagliatesta, P.; Lorecchio, C.; Stella, L.; Kaciulis, S.; Soltani, P.; Placidi, E.; Pizzoferrato, R. Discriminating between Different Heavy Metal Ions with Fullerene-Derived Nanoparticles. Sensors 2018, 18. doi:10.3390/s18051496.

12. Scarpellini, D.; Paoloni, S.; Medaglia, P.; Pizzoferrato, R.; Orsini, A.; Falconi, C. Structural and optical properties of dense vertically aligned $\mathrm{ZnO}$ nanorods grown onto silver and gold thin films by galvanic effect with iron contamination. Materials Research Bulletin 2015, 65, 231-237. doi:https://doi.org/10.1016/j.materresbull.2015.01.059.

13. Frisch, M.J.; Trucks, G.W.; Schlegel, H.B.; Scuseria, G.E.; Robb, M.A.; Cheeseman, J.R.; Scalmani, G.; Barone, V.; Petersson, G.A.; Nakatsuji, H.; Li, X.; Caricato, M.; Marenich, A.V.; Bloino, J.; Janesko, B.G.; Gomperts, R.; Mennucci, B.; Hratchian, H.P.; Ortiz, J.V.; 
Izmaylov, A.F.; Sonnenberg, J.L.; Williams-Young, D.; Ding, F.; Lipparini, F.; Egidi, F.; Goings, J.; Peng, B.; Petrone, A.; Henderson, T.; Ranasinghe, D.; Zakrzewski, V.G.; Gao, J.; Rega, N.; Zheng, G.; Liang, W.; Hada, M.; Ehara, M.; Toyota, K.; Fukuda, R.; Hasegawa, J.; Ishida, M.; Nakajima, T.; Honda, Y.; Kitao, O.; Nakai, H.; Vreven, T.; Throssell, K.; Montgomery, Jr., J.A.; Peralta, J.E.; Ogliaro, F.; Bearpark, M.J.; Heyd, J.J.; Brothers, E.N.; Kudin, K.N.; Staroverov, V.N.; Keith, T.A.; Kobayashi, R.; Normand, J.; Raghavachari, K.; Rendell, A.P.; Burant, J.C.; Iyengar, S.S.; Tomasi, J.; Cossi, M.; Millam, J.M.; Klene, M.; Adamo, C.; Cammi, R.; Ochterski, J.W.; Martin, R.L.; Morokuma, K.; Farkas, O.; Foresman, J.B.; Fox, D.J. Gaussian 16 Revision A.03, 2016. Gaussian Inc. Wallingford CT.

14. Dennington, R.; Keith, T.A.; Millam, J.M. GaussView Version 6, 2019. Semichem Inc. Shawnee Mission KS.

15. Chai, J.D.; Head-Gordon, M. Long-range corrected hybrid density functionals with damped atom-atom dispersion corrections. Phys. Chem. Chem. Phys. 2008, 10, 6615-6620. doi:10.1039/B810189B.

16. Schlegel, H.B. Estimating the hessian for gradient-type geometry optimizations. Theoret. Chim. Acta 1984, 66, 336-340. doi:10.1007/BF00554788.

17. Mennucci, B.; Cancés, E.; Tomasi, J. Evaluation of Solvent Effects in Isotropic and Anisotropic Dielectrics and in Ionic Solutions with a Unified Integral Equation Method: Theoretical Bases, Computational Implementation, and Numerical Applications. J. Phys. Chem. B 1997, 101, 10506-10517, [https://doi.org/10.1021/jp971959k]. doi:10.1021/jp971959k.

18. Adamo, C.; Jacquemin, D. The calculations of excited-state properties with Time-Dependent Density Functional Theory. Chem. Soc. Rev. 2013, 42, 845-856. doi:10.1039/C2CS35394F.

19. Martin, R.L. Natural transition orbitals. J. Chem. Phys. 2003, 118, 4775-4777, [https://doi.org/10.1063/1.1558471]. doi:10.1063/1.1558471. 